\title{
Prevalence and genetic diversity of Enterocytozoon bieneusi in sheep in China
}

\author{
Heng Yang ${ }^{1 \dagger}$, Rongsheng $\mathrm{Mi}^{1 \dagger}$, Long Cheng ${ }^{1}$, Yan Huang ${ }^{1}$, Rui $\mathrm{An}^{2}$, Yehua Zhang ${ }^{1}$, Haiyan Jia ${ }^{1}$, Xiaoli Zhang ${ }^{1}$, \\ Xu Wang ${ }^{1}$, Xiangan $\operatorname{Han}^{1}$ and Zhaoguo Chen ${ }^{1 *}$
}

\begin{abstract}
Background: Enterocytozoon bieneusi is a common species of microsporidia that not only influences human health but also threatens animal productive performance and value. However, there have been no systematic studies of the prevalence of E. bieneusi in sheep in China.

Results: A total of 953 fecal specimens were collected from sheep from 11 provinces across five regions of China and analyzed for E. bieneusi by nested PCR targeting the ribosomal internal transcribed spacer (ITS). Enterocytozoon bieneusi infections were detected in four regions, with an overall infection rate of 20.4\% (194/953). The highest infection rate was detected in pre-weaned lambs (25.0\%), followed by post-weaned lambs (22.2\%) and adult sheep (14.6\%). Enterocytozoon bieneusi was found in nine of the 11 tested provinces, with infection rates between 2.9-51. 7\%. Eleven genotypes were identified based on ITS analysis, including seven known genotypes (BEB6, CHG1, CHG3, CHS7, CHS8, COS-I and NESH5) and four novel genotypes (CHHLS1, CHHLS2, CHNXS1 and CHXJS1). All 11 genotypes were clustered into group 2, and the zoonotic genotype BEB6 was the dominant genotype $(n=129,66.5 \%)$ in sheep.

Conclusion: The prevalence of $E$. bieneusi was studied in five regions representing most areas where sheep are bred in $C h i n a$. This is the first report of E. bieneusi infection in sheep for seven Chinese provinces. Geographical differences were detected in the distribution of E. bieneusi genotypes, but no differences were found among sheep in different age groups. The zoonotic genotype BEB6 was the dominant genotype, indicating that sheep are a potential source of zoonotic microsporidiosis in China. These results improve our knowledge of the epidemiology of E. bieneusi in sheep in China.
\end{abstract}

Keywords: Enterocytozoon bieneusi, Sheep, Prevalence, Genotyping, China

\section{Background}

Microsporidia are obligate intracellular eukaryotic parasites with a wide range of hosts that includes arthropods, birds, mammals and humans $[1,2]$. To date, more than 1300 microsporidian species belonging to 150 genera have been reported [3], including at least 14 microsporidian species belonging to eight families that have been reported to infect humans. The most common species, Enterocytozoon bieneusi $[4,5]$, can cause severe diarrhea in immunocompromised humans and animals, and

\footnotetext{
* Correspondence: zhaoguochen@shvri.ac.cn

${ }^{\dagger}$ Heng Yang and Rongsheng Mi contributed equally to this work.

${ }^{1}$ Key Laboratory of Animal Parasitology of Ministry of Agriculture, Laboratory

of Quality and Safety Risk Assessment for Animal Products on Biohazards

(Shanghai) of Ministry of Agriculture, Shanghai Veterinary Research Institute,

Chinese Academy of Agricultural Sciences, Shanghai 200241, China

Full list of author information is available at the end of the article
}

zoonotic genotypes from domestic animals may be a threat to public health $[1,6,7]$.

Enterocytozoon bieneusi has frequently been reported in domestic animals and wildlife all over the world [7]. Because it is difficult to distinguish E. bieneusi spores using microscopy, and no sophisticated culture approaches have been developed in vitro, molecular methods have been widely used for $E$. bieneusi detection [8]. To date, polymorphisms in the ribosomal internal transcribed spacer (ITS) of the rRNA gene have been widely used for E. bieneusi genotyping, and more than 200 E. bieneusi genotypes have been reported in humans and animals $[8,9]$. When analyzed in combination with phylogeny, these genotypes can be grouped into several genetically isolated clusters [10].

(C) The Author(s). 2018 Open Access This article is distributed under the terms of the Creative Commons Attribution 4.0 International License (http://creativecommons.org/licenses/by/4.0/), which permits unrestricted use, distribution, and reproduction in any medium, provided you give appropriate credit to the original author(s) and the source, provide a link to the Creative Commons license, and indicate if changes were made. The Creative Commons Public Domain Dedication waiver (http://creativecommons.org/publicdomain/zero/1.0/) applies to the data made available in this article, unless otherwise stated. 
Group 1 includes zoonotic genotypes that have been reported in humans and animals [11]. Groups 2 to 9 have mainly been reported in animals and wastewater [11, 12], and few genotypes have been detected in humans [8].

In addition to humans, $E$. bieneusi also infects a variety of animals, such as birds, cats, cattle, deer, dogs, donkeys, horses, pigs and wild mammals [5, 7, 13-20]. However, the epidemiology of $E$. bieneusi has rarely been reported in sheep. To date, a few studies have reported E. bieneusi infection in sheep in Brazil [21], China [19, 22-26], Iran [27] and Sweden [28] (Table 1). Several genotypes were identified in these studies, including genotypes D, BEB6, BEB7, EbpC, O, I, Peru6, NESH1-NESH 6, CS-4, CM7, COS-I-COS-VII and CHS1-CHS12 [7, 22, 23, 25]. Among these, genotypes BEB6, D, EbpC, O, I and Peru6 have been reported in humans $[8,21]$. BEB6 is the most prevalent genotype detected in sheep in Brazil and China [21, 23-25].

In China, E. bieneusi infections have been reported in sheep in five provinces, including Heilongjiang, Henan, Inner Mongolia, Liaoning and Qinghai Province, with infection rates ranging between 4.4-69.3\% [19, 22-26] (Table 1). At the end of 2016, China had the largest number of sheep in the world (http://www.fao.org/faostat/ en/\#data/QA), with most being bred in northern areas. However, there have been no systematic studies of E. bieneusi infection in sheep in China. Therefore, in order to determine the epidemiology and genetic diversity of $E$. bieneusi in sheep in China, 11 provinces across five regions that represent most areas where sheep are farmed in China were selected for $E$. bieneusi testing. These results lay a foundation for a better understanding of the epidemiology and genotypic features of $E$. bieneusi in China.

\section{Methods}

\section{Fecal specimen collection}

Between June 2013 and September 2015, a total of 953 fecal samples were collected from sheep in the following 11 provinces in China: Henan Province in central China; Anhui Province, Shandong Province and Shanghai City in eastern China; Beijing City and Inner Mongolia $\mathrm{Au}-$ tonomous Region in northern China, Heilongjiang Province and Jilin Province in northeast China; and Ningxia Hui Autonomous Region, Qinghai Province and Xinjiang Uyghur Autonomous Region in northwest China (Fig. 1). One fecal specimen was collected from each sheep. The three age groups selected for this study were pre-weaned ( $<3$ months) and post-weaned (3-12 months) lambs, and adult sheep ( $>12$ months). Fresh fecal specimens were collected from sheep using sterile gloves, and each specimen's information was recorded, including location, date and age. Specimens were transported to the laboratory at low temperatures for further detection.

\section{Sample preparation and DNA extraction}

Approximately $300 \mathrm{mg}$ of each fecal specimen was transferred to a $50 \mathrm{ml}$ sterile tube, $20 \mathrm{ml}$ of sterile water was added, and the mixture then stirred with a $1 \mathrm{ml}$ pipette tip to loosen the sample. A 200-300 $\mu \mathrm{l}$ sample was used for DNA extraction with a FastDNA SPIN Kit for Soil (MP Biomedicals, Santa Ana, CA, USA) according to the manufacturer's instructions.

\section{PCR amplification}

A 389 bp fragment of the E. bieneusi ITS gene was amplified by nested PCR as previously described by Buckholt et al. [29]. Briefly, a 435 bp PCR product was amplified

Table 1 Prevalence of E. bieneusi and distribution of ITS genotypes in sheep based on published reports

\begin{tabular}{|c|c|c|c|c|c|}
\hline Year & Country & Province (China) & Prevalence (\%) & Genotype (no. of samples) & Reference \\
\hline 2014 & Sweden & & $45.0(49 / 109)$ & $\begin{array}{l}\mathrm{BEB} 6 \text { (32), OEB1 (6), OEB2 (2), BEB6 + OEB1 (4), } \\
\mathrm{BEB} 6 \text { + OEB2 (4), NDb (1) }\end{array}$ & [28] \\
\hline 2016 & Brazil & & $19.2(24 / 125)$ & $\operatorname{BEB6}(11), \operatorname{BEB} 7$ (8), I (2), BEB18 (1), BEB19 (1), LW1(1) & [21] \\
\hline 2014 & China & Heilongjiang & $4.4(2 / 45)$ & BEB6 (2) & [19] \\
\hline 2015 & China & Heilongjiang & $13.9(68 / 489)$ & $\begin{array}{l}\text { BEB6 (28), CM7 (3), CS-4 (4), BEB6/CM7a (5), } \\
\text { BEB6/OEB1a (5), BEB6/NESH4a (3), OEB1(3), } \\
\text { BEB6/NESH6a (1), CS-4/EbpCa (1), NESH1(1), } \\
\text { NESH2 (1), NESH3 (1), NESH5 (1), }\end{array}$ & [22] \\
\hline 2015 & China & Heilongjiang & $22.5(31 / 138)$ & $\begin{array}{l}\text { BEB6 (12), Peru6 (5), D (4), O (3), COS-I to } \\
\text { COS-VII (one each), }\end{array}$ & [23] \\
\hline 2015 & China & Inner Mongolia & $69.3(260 / 375)$ & BEB6 (237), CM7 (23) & [24] \\
\hline \multirow[t]{3}{*}{2016} & China & Henan & $51.9(161 / 310)$ & $\begin{array}{l}\text { BEB6 (53), COS-I (12), CM4 (1), CHG3 (5), CHS3 (2), } \\
\text { CHS4 (1), CHS5 (1), CHS6 (1), CHS10 (1), CHS12 (1) }\end{array}$ & [25] \\
\hline & & Liaoning & $9.4(6 / 64)$ & BEB6 (3) & \\
\hline & & Heilongjiang & $25.0(10 / 40)$ & $\begin{array}{l}\text { BEB6 (4), COS-I (2), CHS7 (1), CHS8 (1), } \\
\text { CHS9 (1), CHS11 (1) }\end{array}$ & \\
\hline 2018 & China & Qinghai & $23.4(73 / 312)$ & $\begin{array}{l}\text { BEB6 (31), COS-I (25), NESH5 (11), CHS17 (2), } \\
\text { CHS13 (1), CHS14 (1), CHS15 (1), CHS16 (1) }\end{array}$ & [26] \\
\hline
\end{tabular}




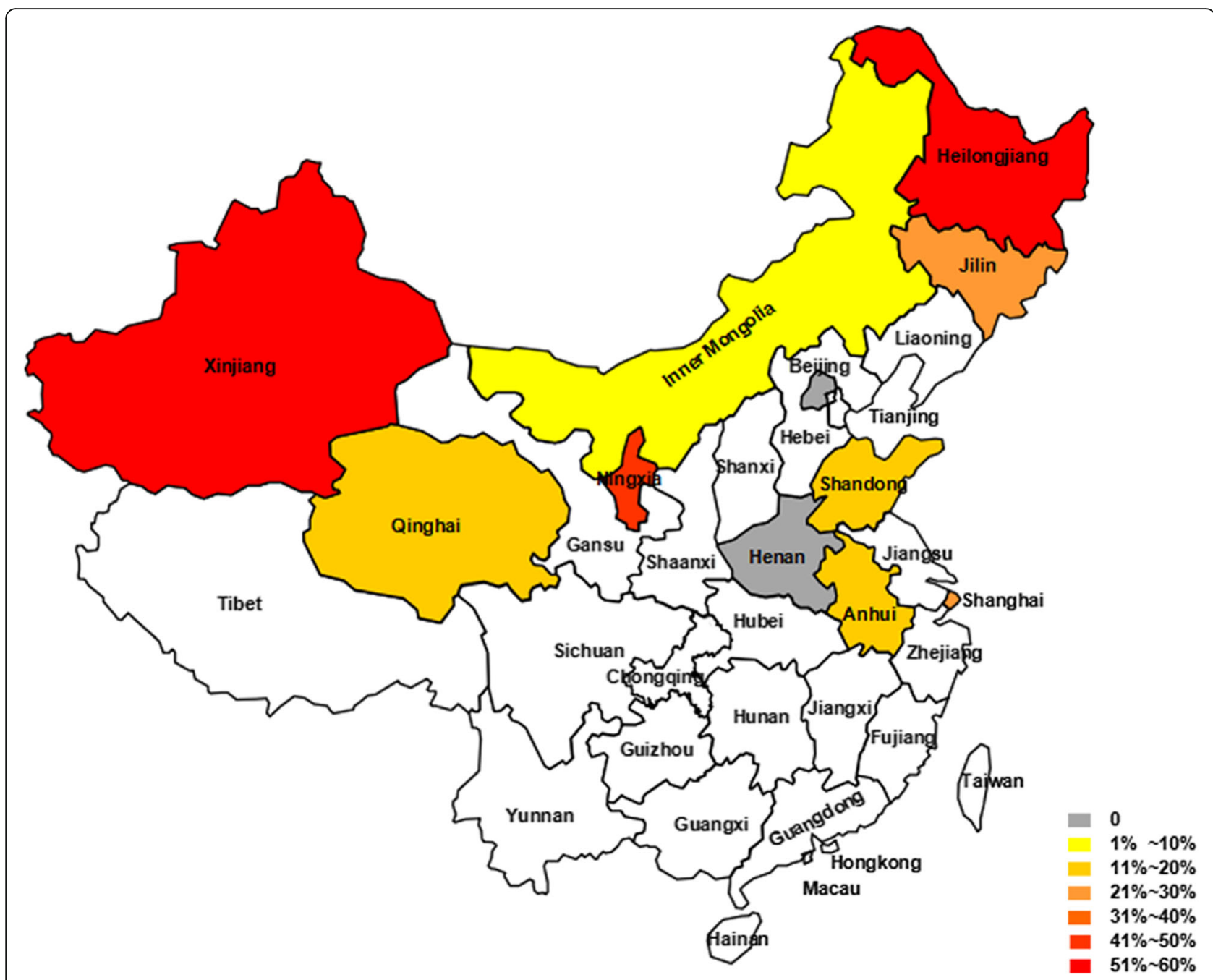

Fig. 1 Geographical map of sampling provinces included in this study. The map was generated using Microsoft Office PowerPoint 2016 software

using the primers EBITS3 (5'-GGT CAT AGG GAT GAA GAG-3') and EBITS4 (5'-TTC GAG TTC TTT CGC GCT C-3') for the primary PCR, and a 389 bp PCR product was amplified using the primers EBITS1 (5'-GCT CTG AAT ATC TAT GGC T-3') and EBITS2.4 (5'-ATC GCC GAC GGA TCC AAG TG-3') for the secondary PCR. Positive and negative controls were included in each PCR reaction. Ex Taq DNA Polymerase (TaKaRa Bio Inc., Beijing, China) was used for PCR amplifications. The secondary PCR products were examined by gel electrophoresis on a $1.2 \%$ agarose gel containing $4 \mathrm{~S}$ Green Plus Nucleic Acid Stain (Sangon Biotech, Shanghai, China).

\section{Sequence analysis and phylogenetic construction}

Positive samples from the second round of PCR gel electrophoresis were sequenced and analyzed using the BLAST program (http://blast.ncbi.nlm.nih.gov/Blast.cgi). Phylogenetic analysis of $E$. bieneusi was performed using
MEGA 7.0 software [30]. All positive samples were sequenced twice, and novel genotypes were designated as previously described by Santín \& Fayer [8]. Briefly, all sequences obtained in the present study were analyzed using the BLAST program to determine the E. bieneusi genotype based on $243 \mathrm{bp}$ of the ITS gene region. Sequences that differed from published genotypes were considered new genotypes.

\section{Statistical analysis}

Differences in infection rates between different regions, provinces and ages were assessed using a Chi-square test with SPSS Version 21.0 software (IBM Corp., Armonk, NY, USA). Differences were considered statistically significant when $P<0.05$. Prevalence rates and 95\% confidence intervals $(\mathrm{CI})$ were also determined. Unique ITS sequences of $E$. bieneusi were submitted to the GenBank database under the accession numbers MH432644-MH432647. 


\section{Results}

Overall prevalence and genotypic features of $E$. bieneusi in sheep

The overall prevalence of $E$. bieneusi infection in sheep in this study was 20.4\% (194/953; 95\% CI: 17.8-23.1\%). All positive samples were sequenced for E. bieneusi genotype determination based on BLAST analyses. Eleven $E$. bieneusi genotypes were identified, including seven previously described genotypes (BEB6, CHG1, CHG3, CHS7, CHS8, COS-I and NESH5) and four novel genotypes (CHHLJS1, CHHLJS2, CHNXS1 and CHXJS1) (Table 2).

Overall, BEB6 $(n=129)$ was the dominant genotype, followed by CHS8 $(n=32)$ and CHG1 $(n=14)$. All other genotypes were present in less than five specimens (Table 2).

\section{Prevalence of $E$. bieneusi in sheep in different regions of China}

Of the five regions tested for $E$. bieneusi infections in sheep, northeast China had the highest infection rate (38.5\%, 50/130; 95\% CI: 30.1-47.4\%), followed by northwest China (28.0\%, 83/296; 95\% CI: 23.0-33.5\%), eastern China (17.8\%, 58/326; 95\% CI: 13.8-22.4\%) and northern China (1.8\%, 3/166; 95\% CI: 0.4-5.2\%). Enterocytozoon bieneusi infection was not detected in central China (Table 2). A significant difference was observed between different regions $\left(\chi^{2}=82.562, d f=4, P<0.0001\right)$. The most common genotype, BEB6, was detected in all $E$. bieneusi-positive regions. In northwest China, we found high prevalence rates of both BEB6 $(57.8 \%, 48 / 83)$ and CHS8 (38.6\%, 32/83) (Table 2).

\section{Prevalence of $E$. bieneusi in sheep in different provinces in China}

Of the 11 provinces tested for E. bieneusi in sheep, the highest infection rate was detected in Heilongjiang (51.7\%, 31/60; 95\% CI: 38.4-64.8\%), followed by Ningxia (47.1\%, 57/121; 95\% CI: 38.0-56.4\%), Jilin (27.1\%, 19/70; 95\% CI: 17.2-39.1\%), Shanghai (23.7\%, 36/152; 95\% CI: 17.2-31.3\%), Xinjiang (19.2\%, 19/99; 95\% CI: $12.0-$ $28.3 \%)$, Shandong (13.1\%, 16/122; 95\% CI: 7.7-20.4\%),
Anhui (11.5\%, 6/52; 95\% CI: 4.4-23.4\%), Qinghai (9.2\%, 7/76; 95\% CI: 3.8-18.1\%) and Inner Mongolia (2.9\%, 3/ 102; 95\% CI: 0.6-8.4\%). Enterocytozoon bieneusi infection was not detected in Beijing (0/64; 95\% CI: 0-5.6\%) or Henan (0/35; 95\% CI: 0-10.0\%) (Table 3). Statistical analysis showed significant differences in infection rates of E. bieneusi among the different provinces $\left(\chi^{2}=149.446\right.$, $d f=10, P<0.0001)$.

The distribution of $E$. bieneusi genotypes differed among the different provinces. The most common genotype, BEB6, was detected in Heilongjiang (21/31), Inner Mongolia (3/3), Jilin (19/19), Qinghai (6/7), Shanghai $(35 / 36)$ and Xinjiang (18/19). In contrast, CHG1 (11/16) and CHS8 (32/57) were the dominant genotypes in Shandong and Ningxia, respectively. Four novel genotypes were identified in Heilongjiang $(n=2)$, Ningxia $(n=1)$ and Xinjiang $(n=1)$. More than two genotypes were detected in most provinces, with the exception of Jilin and Inner Mongolia, where only one genotype (BEB6) was found (Table 3).

Prevalence of E. bieneusi in different age groups of sheep Enterocytozoon bieneusi infections were detected in all three age groups. The highest prevalence rate was detected in pre-weaned lambs $(25.0 \%, 73 / 292 ; 95 \% \mathrm{CI}$ : 20.1-30.4\%), followed by post-weaned lambs $(22.2 \%, 72 /$ 325; 95\% CI: $17.8-27.1 \%)$ and adult sheep (14.6\%, 49/ 336; $95 \%$ CI: $11.0-18.8 \%)$, with statistically significant differences among the age groups $\left(\chi^{2}=11.438, d f=2\right.$, $P=0.003)$. More than six genotypes were identified in all age groups, and BEB6 was the most common genotype detected in each group (Table 4).

At the province level, infection rates of E. bieneusi differed by age group. The most susceptible age group was pre-weaned lambs, and $E$. bieneusi was detected in sheep in this age group in most provinces, including Inner Mongolia (8.1\%, 3/31; 95\% CI: 1.7-21.9\%), Jilin (41.7\%, 10/24; 95\% CI: 22.1-63.4\%), Ningxia (57.5\%, 23/40; 95\% CI: 40.9-73.0\%), Qinghai (12.5\%, 3/24; 95\% CI: 2.7-32.4\%), Shanghai (30.0\%, 6/20; 95\% CI: 11.9-54.3\%) and Xinjiang (38.2\%, 13/34; 95\% CI: 22.2-56.4\%). However, in two

Table 2 Prevalence and genotype distribution of E. bieneusi in sheep from different regions of China

\begin{tabular}{lllll}
\hline Region & No. of specimens & No. positive (\%) & $95 \%$ Cl & Genotype (no. of samples) \\
\hline Central China & 35 & $0^{d}$ & $0-10.0$ & \\
Eastern China & 326 & $58(17.8)^{\mathrm{c}}$ & $13.8-22.4$ & BEB6 (38), CHG1 (14), CHG3 (5), COS-I (1) \\
Northern China & 166 & $3(1.8)^{\mathrm{d}}$ & $0.4-5.2$ & BEB6 (3) \\
Northeast China & 130 & $50(38.5)^{\mathrm{a}}$ & $30.1-47.4$ & BEB6 (40), CHS7 (3), CHHLS1 (3), CHHLS2 (2), COS-I (2) \\
Northwest China & 296 & $83(28.0)^{\mathrm{b}}$ & $23.0-33.5$ & BEB6 (48), CHS8 (32), CHNXS1 (1), CHXJS1 (1), NESH5 (1) \\
Total & 953 & $194(20.4)$ & $17.8-23.1$ & BEB6 (129), CHS8 (32), CHG1 (14), CHG3 (5), CHS7 (3), \\
& & & COS-I (3), CHHLS1 (3), CHHLS2 (2), CHNXS1 (1),
\end{tabular}

Note: Different superscript letters within columns represent significant differences between groups $(P<0.05)$. Novel genotypes identified in this study are indicated in bold 
Table 3 Prevalence and genotype distribution of E. bieneusi in sheep from different provinces of China

\begin{tabular}{llllll}
\hline Region & Province & No. of specimens & No. positive (\%) & $95 \% \mathrm{Cl}$ & Genotype (no. of samples) \\
\hline Central China & Henan & 35 & $0^{\mathrm{e}}$ & $0-10.0$ & \\
Eastern China & Anhui & 52 & $6(11.5)^{\mathrm{c}}$ & $4.4-23.4$ & CHG1 (3), CHG3 (3) \\
& Shandong & 122 & $16(13.1)^{\mathrm{c}}$ & $7.7-20.4$ & BEB6 (3), CHG1 (11), CHG3 (2) \\
& Shanghai & 152 & $36(23.7)^{\mathrm{b}}$ & $17.2-31.3$ & BEB6 (35), COS-I (1) \\
Northern China & Beijing & 64 & $0^{\mathrm{e}}$ & $0-5.6$ & BEB6 (3) \\
& Inner Mongolia & 102 & $3(2.9)^{\mathrm{de}}$ & $0.6-8.4$ & BEB6 (21), CHS7 (3), CHHLS1 (3), \\
Northeast China & Heilongjiang & 60 & $31(51.7)^{\mathrm{a}}$ & $38.4-64.8$ & COS-I (2), CHHLJS2 (2) \\
& Jilin & 70 & $19(27.1)^{\mathrm{b}}$ & $17.2-39.1$ & BEB6 (19) \\
Northwest China & Ningxia & 121 & $57(47.1)^{\mathrm{a}}$ & $38.0-56.4$ & BEB6 (24), CHS8 (32), CHNXS1 (1) \\
& Qinghai & 76 & $7(9.2)^{\mathrm{cd}}$ & $3.8-18.1$ & BEB6 (6), NESH5 (1) \\
Total & Xinjiang & 99 & $19(19.2)^{\mathrm{bc}}$ & $12.0-28.3$ & BEB6 (18), CHXJS1 (1)
\end{tabular}

Note: Different superscript letters within columns represent significant differences between groups $(P<0.05)$

provinces, Anhui $(13.6 \%, 3 / 22 ; 95 \%$ CI: $2.9-34.9 \%)$ and Heilongiiang (80.0\%, 16/20; 95\% CI: 56.3-94.3\%), postweaned lambs had the highest prevalence rate, and in Shandong Province (27.8\%, 10/36; 95\% CI: $14.2-45.2 \%)$ the group with the highest prevalence rate was adult sheep. With the exception of Anhui Province, BEB6 was detected in each group in all provinces (Table 5).

\section{Phylogenetic analysis}

Phylogenetic trees were constructed using the novel and known genotypes based on ITS nucleotide sequences as shown in Fig. 2. The results indicated that all genotypes detected in this study belonged to group 2 .

\section{Discussion}

In the present study, an investigation of E. bieneusi was conducted in 11 provinces, which included most areas where sheep are farmed in China. Enterocytozoon bieneusi infection was detected in nine provinces, suggesting that $E$. bieneusi is widespread in sheep in China. Compared to studies that used the same testing method, the overall prevalence of $E$. bieneusi in sheep in this study was $20.4 \%$, which is similar to previous reports of $E$. bieneusi in sheep in Brazil (19.2\%) [21], but lower than prevalence rates reported in Sweden (68.1\%) [28]. The prevalence rate in this study was also higher than a previous report from Iran (10.0\%), which was based on the SSU rRNA gene [27] (Table 1). Additionally, the prevalence rate of $E$. bieneusi in Heilongjiang in the present study $(51.7 \%)$ was higher than the previously reported rate of $22.5 \%$ found using the same method [23]. However, compared to studies that used different ITS primers, prevalence rates in the present study in Henan (0\%), Inner Mongolia (2.9\%) and Qinghai (9.2\%) were lower than previous reports in Henan (51.9\%) [25], Inner Mongolia (69.3\%) [24] and Qinghai (23.4\%) [26], but higher than previous reports in Heilongjiang (4.4, 13.9 and $25.0 \%)$ [19, 22, 25]. The differences between our study and previous studies may be due to differences in detection methods, sampling sites, sampling seasons, sheep age groups or sheep densities. Prevalence rates of $E$. bieneusi in the other provinces, including Ningxia (47.1\%), Jilin (27.1\%), Shanghai (23.7\%), Shandong (13.1\%), Xinjiang (19.2\%), Anhui (11.5\%) and Beijing (0\%), have not been previously reported.

In the present study, the prevalence of $E$. bieneusi was assessed in five regions, which include most areas where sheep are bred in China. We did not obtain samples from southeast or southwest China, mainly because goats are the dominant livestock in these areas. The

Table 4 Prevalence and ITS genotype distribution of E. bieneusi in different age groups of sheep

\begin{tabular}{|c|c|c|c|c|}
\hline Age & No. of specimens & No. positive (\%) & $95 \% \mathrm{Cl}$ & Genotype (no. of samples) \\
\hline Pre-weaned lambs & 292 & $73(25.0)^{\mathrm{a}}$ & $20.1-30.4$ & $\begin{array}{l}\text { BEB6 (54), CHS8 (12), CHG3 (2), COS-I (1), CHG1 (1), } \\
\text { CHHLJS2 (1), CHNXS1 (1), CHXJS1 (1) }\end{array}$ \\
\hline Post-weaned lambs & 325 & $72(22.2)^{\mathrm{a}}$ & $17.8-27.1$ & $\begin{array}{l}\text { BEB6 (47), CHS8 (11), CHS7 (2), COS-I (2), CHG1 (2), } \\
\text { CHG3 (2), CHHLJS1 (2), CHSDS2 (2), NESH5 (1), CHHLJS2 (1) }\end{array}$ \\
\hline Adult sheep & 336 & $49(14.6)^{b}$ & $11.0-18.8$ & BEB6 (28), CHS8 (9), CHG1 (9), CHS7 (1), CHG3 (1), CHHLJS1 (1) \\
\hline Total & 953 & $194(20.4)$ & $17.8-23.1$ & \\
\hline
\end{tabular}

Note: Different superscript letters within columns represent significant differences between groups $(P<0.05)$ 
Table 5 Prevalence and ITS genotype distribution of E. bieneusi in different age groups from different provinces

\begin{tabular}{|c|c|c|c|c|c|}
\hline Province & Age & No. of specimens & No. positive (\%) & $95 \% \mathrm{Cl}$ & Genotype (no. of samples) \\
\hline \multirow[t]{3}{*}{ Anhui } & Pre-weaned lambs & 16 & $2(12.5)$ & $1.6-38.4$ & CHG3 (2) \\
\hline & Post-weaned lambs & 22 & $3(13.6)$ & $2.9-34.9$ & CHG1 (2), CHG3 (1) \\
\hline & Adult sheep & 14 & $1(7.1)$ & $0.2-33.9$ & CHG1 (1) \\
\hline \multirow[t]{3}{*}{ Beijing } & Pre-weaned lambs & 20 & 0 & $0-16.8$ & \\
\hline & Post-weaned lambs & 22 & 0 & $0-15.4$ & \\
\hline & Adult sheep & 22 & 0 & $0-15.4$ & \\
\hline \multirow[t]{3}{*}{ Heilongjiang } & Pre-weaned lambs & 20 & $12(60.0)$ & $36.1-80.9$ & BEB6 (11), CHHLJS2 (1) \\
\hline & Post-weaned lambs & 20 & $16(80.0)$ & $56.3-94.3$ & $\begin{array}{l}\text { BEB6 (9), CHS7 (2), COS-I (2), CHHLJS1 (2), } \\
\text { CHHLIS2 (1) }\end{array}$ \\
\hline & Adult sheep & 20 & $3(15.0)$ & $3.2-37.9$ & BEB6 (1), CHS7 (1), CHHLJS1 (1) \\
\hline Henan & Adult sheep & 35 & 0 & $0-10.0$ & \\
\hline \multirow[t]{3}{*}{ Inner Mongolia } & Pre-weaned lambs & 37 & $3(8.1)$ & $1.7-21.9$ & BEB6 (3) \\
\hline & Post-weaned lambs & 35 & 0 & $0-10.0$ & \\
\hline & Adult sheep & 30 & 0 & $0-11.6$ & \\
\hline \multirow[t]{3}{*}{ Jilin } & Pre-weaned lambs & 24 & $10(41.7)$ & $22.1-63.4$ & BEB6 (10) \\
\hline & Post-weaned lambs & 25 & $6(24.0)$ & $9.4-45.1$ & BEB6 (6) \\
\hline & Adult sheep & 21 & $3(14.3)$ & $3.1-36.3$ & BEB6 (3) \\
\hline \multirow[t]{3}{*}{ Ningxia } & Pre-weaned lambs & 40 & $23(57.5)$ & $40.9-73.0$ & CHS8 (12), BEB6 (10), CHNXS1 (1) \\
\hline & Post-weaned lambs & 40 & $17(42.5)$ & $27.0-59.1$ & CHS8 (11), BEB6 (6) \\
\hline & Adult sheep & 41 & $17(41.5)$ & $26.3-57.9$ & CHS8 (9), BEB6 (8) \\
\hline \multirow[t]{3}{*}{ Qinghai } & Pre-weaned lambs & 24 & $3(12.5)$ & $2.7-32.4$ & BEB6 (3) \\
\hline & Post-weaned lambs & 32 & $3(9.4)$ & $2.0-25.0$ & BEB6 (2), NESH5 (1) \\
\hline & Adult sheep & 20 & $1(5.0)$ & $0.1-24.9$ & BEB6 (1) \\
\hline \multirow[t]{3}{*}{ Shandong } & Pre-weaned lambs & 57 & $1(1.8)$ & $0-9.4$ & CHG1 (1) \\
\hline & Post-weaned lambs & 29 & $5(17.2)$ & $5.9-35.8$ & BEB6 (2), CHG1 (2), CHG3 (1) \\
\hline & Adult sheep & 36 & $10(27.8)$ & $14.2-45.2$ & CHG1 (8), BEB6 (1), CHG3 (1) \\
\hline \multirow[t]{3}{*}{ Shanghai } & Pre-weaned lambs & 20 & $6(30.0)$ & $11.9-54.3$ & BEB6 (5), COS-I (1) \\
\hline & Post-weaned lambs & 64 & $17(26.6)$ & $16.3-39.1$ & BEB6 (17) \\
\hline & Adult sheep & 68 & $13(19.1)$ & $10.6-30.5$ & BEB6 (13) \\
\hline \multirow[t]{3}{*}{ Xinjiang } & Pre-weaned lambs & 34 & $13(38.2)$ & $22.2-56.4$ & BEB6 (12), CHXJS1 (1) \\
\hline & Post-weaned lambs & 36 & $5(13.9)$ & $4.7-29.5$ & BEB6 (5) \\
\hline & Adult sheep & 29 & $1(3.5)$ & $0.1-17.8$ & BEB6 (1) \\
\hline
\end{tabular}

prevalence of E. bieneusi in northeast China (38.5\%) was higher than previously reported (13.9\%) [22]. This may be due to differences in sampling sites or sheep age groups. In the present study, we did not detect E. bieneusi infection in central China, possibly because only adult sheep were tested in this area (Table 5). Similar results were also reported in China and Sweden [19, 28], where $E$. bieneusi infections were not detected in adult sheep. Therefore, future studies need to increase the number of specimens collected in this area.

In the present study, a higher prevalence of $E$. bieneusi was detected in lambs (23.5\%) than in adults (14.6\%), which is consistent with many previous studies in Brazil and China [21, 22, 24-26]. The prevalence of E. bieneusi in lambs under one year of age was $23.5 \%$, which is similar to the findings of a previous study in Heilongiiang (18.2\%) [22], but lower than reported in previous studies in other parts of China [25, 26] and in Sweden [28]. The prevalence in pre-weaned lambs $(25.0 \%)$ was also similar to a study by $\mathrm{Li}$ et al. [19] in Heilongjiang (20.0\%) but was significantly lower than previous reports by $\mathrm{Ye}$ et al. [24] in Inner Mongolia (77.8\%). We also observed differences in adult sheep between this study and previous studies; the reported prevalence in this study (14.6\%) was higher than that reported in Brazil (11.1\%) by Fiuza et al. [21] and in China (8.7\%) by Jiang et al. [22], but was lower than the prevalence reported in Inner Mongolia (62.9\%) [24] and in Qinghai (22.7\%) [26]. Another report also 


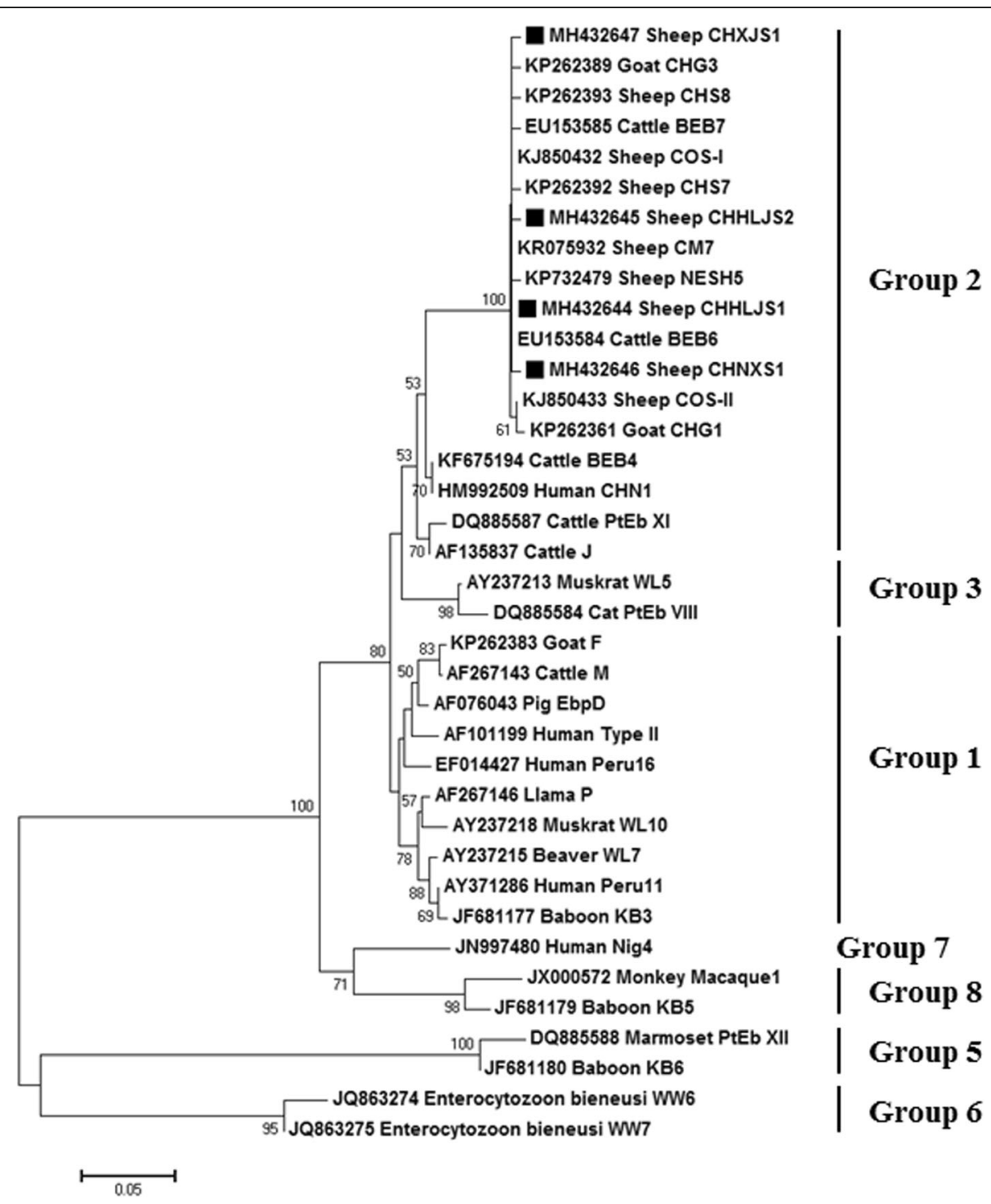

Fig. 2 Phylogenetic analysis of E. bieneusi based on ITS gene sequences. The evolutionary history was inferred using the neighbor-joining method and evolutionary analyses were conducted in MEGA 7.0. Bootstrap values $>50 \%$ are shown. Filled squares indicate the novel genotypes obtained in the present study

found a higher prevalence of E. bieneusi (39.4\%) in adult sheep in Liaoning, Henan and Heilongjiang [25]. In contrast, two studies reported no E. bieneusi infection in adult sheep in Sweden [28] or Heilongjiang [19]. From these results, it seems that sheep age can affect the E. bieneusi infection rate in different regions.

The first reported $E$. bieneusi genotype, BEB6, was described in cattle in the USA [14]. Since then, this genotype has become common in ruminants, including sheep [19, 21-26, 28], goats [31], golden takins [32], deer [20], sika deer [20] and alpacas [33]. In addition, this genotype has also been found in humans [34], cats [15], rhesus macaques [16], yaks [26], horses [35], ducks and geese [36], as well as in urban wastewater [37, 38]. In this study, BEB6 was the most prevalent genotype (66.5\%) and was detected in all provinces where E. bieneusi infection was found. These results are consistent with previous reports in the same provinces of China, including Heilongjiang [19, 22, 23, 25], Inner Mongolia [24] and Qinghai [26]. Other studies have also reported that BEB6 is the most common genotype in sheep in Sweden [28] and Brazil [21]. In China, BEB6 was also reported to be the most prevalent genotype in other provinces, such as Liaoning and Henan [25]. According to these results, we suggest that BEB6 is a dominant genotype with a widespread geographical distribution in sheep.

In this study, we identified five genotypes (CHG3, CHS7, CHS8, COS-I and NESH5) that were previously 
reported in sheep in China. CHG3 was previously detected in Henan [25]; CHS7 and CHS8 were previously detected in Heilongjiang [25]; COS-I was previously reported in Heilongjiang [23, 25], Henan [25] and Qinghai [26]; and NESH5 was previously found in Heilongjiang [22] and Qinghai [26]. Similar to these studies, we also found CHS7 and COS-I in Heilongjiang and NESH5 in Qinghai. However, we did not detect genotypes that had been previously reported in some provinces, such as genotype CM7, which was reported in Inner Mongolia and Heilongjiang [22, 24]. Although genotype CHG1 was previously reported in goats in Chongqing, Henan, Shaanxi and Yunnan [25], this study is the first report of this genotype in sheep. Phylogenetic analysis showed that all genotypes belonged to group 2. Although it has been classified as a cattle-specific group [11], some group 2 genotypes have been found in humans, including genotypes BEB6, I and J.

Based on known E. bieneusi genotypes [8], four novel genotypes were identified in sheep in this study. Compared to the known genotype BEB6, the novel genotypes CHHLJS1, CHNXS1 and CHXJS1 had two substitutions (A to $\mathrm{T}$ at position 155 and $\mathrm{A}$ to $\mathrm{T}$ at position 178), one substitution (A to $G$ at position 135) and one substitution (A to $G$ at positions 181), respectively. Compared to genotype CHS3, CHHLJS1 had one substitution ( $\mathrm{G}$ to A at position 86). Phylogenetic analysis showed that all novel genotypes belonged to group 2 .

According to the results of our study, there were differences in the geographical distribution of ITS genotypes in sheep. Although BEB6 was the most common genotype in most provinces, we found that CHS8 (56.1\%) was the dominant genotype in Ningxia and CHG1 (68.7\%) was the dominant genotype in Shandong. In addition, equal occurrences of CHG1 $(n=3)$ and CHG3 $(n=3)$ were found in Anhui. In most provinces, more than two genotypes were detected. Similar to previous studies, which reported several genotypes in Heilongjiang [22, 23, 25], we found more than five genotypes in this province (Table 1). Differences in the geographical distribution of E. bieneusi genotypes have also been reported in other studies. For example, BEB6 was the only genotype reported in Heilongjiang and Liaoning [19, 25], and only two genotypes (BEB6 and CM7) were previously reported in Inner Mongolia [23]. Moreover, more than five genotypes have been reported in Brazil [21], China [22, 23, 25, 26] and Sweden [28]. However, it seems that the distribution of ITS genotypes was not related to sheep age. In this study, more than six genotypes were detected in all age groups, and the distribution of ITS genotypes was similar between the groups, which is consistent with a previous study in Inner Mongolia [23].

Enterocytozoon bieneusi is an important protozoan parasite that is transmitted via water and food. Humans can become infected with microsporidia through human or animal fecal contamination in soil or water [39, 40]. In China, the E. bieneusi genotype BEB6 has been detected in wastewater from five cities [37, 41]. In the present study, BEB6 was the most prevalent genotype, indicating that sheep may be a source of $E$. bieneusi contamination in wastewater. However, whether E. bieneusi is present in wastewater near farms in these areas is unclear, and more studies are required to further understand the transmission of $E$. bieneusi between sheep and water.

\section{Conclusions}

In this study, we assessed the prevalence and genetic diversity of $E$. bieneusi in sheep from 11 provinces across five regions of China. E. bieneusi was found in nine provinces, suggesting that $E$. bieneusi is widespread in sheep in China. The overall infection rate was $20.4 \%$, and the highest infection rate was detected in pre-weaned lambs. At the province level, the prevalence in different age groups also differed. Eleven genotypes were detected in sheep in this study, including four novel genotypes. The zoonotic genotype BEB6 was the dominant genotype and may pose a potential threat to humans. We also observed geographical differences in the genotypic features of E. bieneusi in sheep, but no differences were found in genotypes among the different age groups. This study covered most areas of China where sheep are bred, and for seven of the provinces this is the first report of E. bieneusi. Therefore, this study increases our understanding of the prevalence and genotypic characterization of $E$. bieneusi in sheep in China.

\section{Abbreviations \\ ITS: Internal transcribed spacer; PCR: Polymerase chain reaction; SSU rRNA: Small subunit ribosomal RNA}

\section{Acknowledgements}

We thank Guodong Mu at Jilin Center for Animal Disease Control and Prevention, Haining Zhou at Ningxia Center for Animal Disease Control and Prevention, Wei Zhu at Tengzhou Animal Husbandry and Veterinary Technology Service Center, and Yibin Hu at Beijing Centre Polytron Technologies Inc. for their help with sample collection. We also thank International Science Editing for editing this manuscript.

\section{Funding}

This study was supported in part by The National Key Research and Development Programme of China (grant no. 2018YFD0502305), National Risk Assessment Project for Quality and Safety of Agricultural Products (grant no. GJFP201800703), Shanghai Agriculture Applied Technology Development Program, China (grant no. G20180110), Technical Standard Programme of Shanghai Science and Technology Commission (grant no. 16DZ0501900) and The Key Technology R \& D Programme of Ningxia Hui Autonomous Region, China (grant no. 201601).

\section{Availability of data and materials}

The datasets supporting the conclusions in this article are included within the article. Sequences were submitted to the GenBank database under the accession numbers MH432644-MH432647.

\section{Authors' contributions}

ZC designed the study and revised the manuscript. HY, RM and LC performed the experiments and drafted the manuscript. YH and RA participated in sample collection. YZ, HJ and XZ participated in DNA extraction and PCR amplification. 
XW and $\mathrm{XH}$ participated in data analysis. All authors read and approved the final manuscript.

\section{Ethics approval and consent to participate}

Faecal samples were collected from sheep farms with the consent of farm owners. All experiments were authorised by the Animal Ethics Committee of the Shanghai Veterinary Research Institute and approved by the Animal Care and Use Committee of the Chinese Academy of Agricultural Sciences. The Animal Ethics Committee approval number was Shvri-sh-2013020042. During the whole experimental process, all laboratory work on the study specimens were covered under the Animal Experimental Protocol of Shanghai Veterinary Research Institute (201008): "Use of animal samples for the determination of zoonotic pathogen".

\section{Consent for publication}

Not applicable.

\section{Competing interests}

The authors declare that they have no competing interests.

\section{Publisher's Note}

Springer Nature remains neutral with regard to jurisdictional claims in published maps and institutional affiliations.

\section{Author details}

${ }^{1}$ Key Laboratory of Animal Parasitology of Ministry of Agriculture, Laboratory of Quality and Safety Risk Assessment for Animal Products on Biohazards (Shanghai) of Ministry of Agriculture, Shanghai Veterinary Research Institute, Chinese Academy of Agricultural Sciences, Shanghai 200241, China. ${ }^{2}$ Animal Husbandry and Veterinary Bureau of Changji Hui Autonomous Prefecture, Xinjiang Uyghur Autonomous Region, Changji 831100, Xinjiang, China.

Received: 6 August 2018 Accepted: 29 October 2018

\section{Published online: 12 November 2018}

\section{References}

1. Didier ES, Weiss LM. Microsporidiosis: current status. Curr Opin Infect Dis. 2006;19:485-92

2. Didier ES. Microsporidiosis: an emerging and opportunistic infection in humans and animals. Acta Trop. 2005:94:61-76.

3. Keeling P. Five questions about microsporidia. PLoS Pathog. 2009;5: e1000489.

4. Mathis A, Weber R, Deplazes P. Zoonotic potential of the microsporidia. Clin Microbiol Rev. 2005;18:423-45.

5. Matos O, Lobo ML, Xiao L. Epidemiology of Enterocytozoon bieneusi infection in humans. J Parasitol Res. 2012;2012:981424.

6. Keeling PJ, Fast NM. Microsporidia: biology and evolution of highly reduced intracellular parasites. Annu Rev Microbiol. 2002;56:93-116.

7. Santín M, Fayer R. Microsporidiosis: Enterocytozoon bieneusi in domesticated and wild animals. Res Vet Sci. 2011;90:363-71.

8. Santín M, Fayer R. Enterocytozoon bieneusi genotype nomenclature based on the internal transcribed spacer sequence: a consensus. J Eukaryot Microbiol. 2009;56:34-8.

9. Santín M, Fayer R. Enterocytozoon bieneusi, Giardia, and Cryptosporidium infecting white-tailed deer. J Eukaryot Microbiol. 2015;62:34-43.

10. Karim MR, Dong H, Li T, Yu F, Li D, Zhang L, et al. Predomination and new genotypes of Enterocytozoon bieneusi in captive nonhuman primates in zoos in China: high genetic diversity and zoonotic significance. PLoS One. 2015;10:e117991.

11. Thellier M, Breton J. Enterocytozoon bieneusi in human and animals, focus on laboratory identification and molecular epidemiology. Parasite. 2008; 15:349-58.

12. Guo Y, Alderisio KA, Yang W, Cama V, Feng Y, Xiao L. Host specificity and source of Enterocytozoon bieneusi genotypes in a drinking source watershed. Appl Environ Microbiol. 2014;80:218-25.

13. Yue DM, Ma JG, Li FC, Hou JL, Zheng WB, Zhao Q, et al. Occurrence of Enterocytozoon bieneusi in donkeys (Equus asinus) in China: a public health concern. Front Microbiol. 2017;8:565.

14. Fayer R, Santín M, Trout JM. Enterocytozoon bieneusi in mature dairy cattle on farms in the eastern United States. Parasitol Res. 2007;102:15-20.
15. Karim MR, Dong H, Yu F, Jian F, Zhang L, Wang R, et al. Genetic diversity in Enterocytozoon bieneusi isolates from dogs and cats in China: host specificity and public health implications. J Clin Microbiol. 2014;52:3297-302.

16. Karim MR, Wang R, Dong H, Zhang L, Li J, Zhang S, et al. Genetic polymorphism and zoonotic potential of Enterocytozoon bieneusi from nonhuman primates in China. Appl Environ Microbiol. 2014;80:1893-8.

17. Li J, Luo N, Wang C, Qi M, Cao J, Cui Z, et al. Occurrence, molecular characterization and predominant genotypes of Enterocytozoon bieneusi in dairy cattle in Henan and Ningxia, China. Parasit Vectors. 2016;9:142.

18. Li W, Tao W, Jiang Y, Diao R, Yang J, Xiao L. Genotypic distribution and phylogenetic characterization of Enterocytozoon bieneusi in diarrheic chickens and pigs in multiple cities, China: potential zoonotic transmission. PLoS One. 2014:9:e108279.

19. Li W, Li Y, Li W, Yang J, Song M, Diao R, et al. Genotypes of Enterocytozoon bieneusi in livestock in China: high prevalence and zoonotic potential. PLoS One. 2014;9:e97623.

20. Zhao W, Zhang W, Wang R, Liu W, Liu A, Yang D, et al. Enterocytozoon bieneusi in sika deer (Cervus nippon) and red deer (Cervus elaphus): deer specificity and zoonotic potential of ITS genotypes. Parasitol Res. 2014;113: 4243-50.

21. Fiuza VRDS, Lopes CWG, Cosendey RIJ, de Oliveira FCR, Fayer R, Santín M. Zoonotic Enterocytozoon bieneusi genotypes found in Brazilian sheep. Res Vet Sci. 2016;107:196-201.

22. Jiang Y, Tao W, Wan Q, Li Q, Yang Y, Lin Y, et al. Zoonotic and potentially host-adapted Enterocytozoon bieneusi genotypes in sheep and cattle in northeast China and an increasing concern about the zoonotic importance of previously considered ruminant-adapted genotypes. Appl Environ Microbiol. 2015;81:3326-35.

23. Zhao W, Zhang W, Yang D, Zhang L, Wang R, Liu A. Prevalence of Enterocytozoon bieneusi and genetic diversity of ITS genotypes in sheep and goats in China. Infect Genet Evol. 2015;32:265-70.

24. Ye J, Xiao L, Wang Y, Guo Y, Roellig DM, Feng Y. Dominance of Giardia duodenalis assemblage $A$ and Enterocytozoon bieneusi genotype BEB6 in sheep in Inner Mongolia, China. Vet Parasitol. 2015;210:235-9.

25. Shi K, Li M, Wang X, Li J, Karim MR, Wang R, et al. Molecular survey of Enterocytozoon bieneusi in sheep and goats in China. Parasit Vectors. 2016:9:23

26. Zhang Q, Cai J, Li P, Wang L, Guo Y, Li C, et al. Enterocytozoon bieneusi genotypes in Tibetan sheep and yaks. Parasitol Res. 2018;117:721-7.

27. Askari Z, Mirjalali H, Mohebali M, Zarei Z, Shojaei S, Rezaeian T, et al. Molecular detection and identification of zoonotic Microsporidia spore in fecal samples of some animals with close-contact to human. Iran J Parasitol. 2015:10:381-8

28. Stensvold CR, Beser J, Ljungström B, Troell K, Lebbad M. Low host-specific Enterocytozoon bieneusi genotype BEB6 is common in Swedish lambs. Vet Parasitol. 2014;205:371-4

29. Buckholt MA, Lee JH, Tzipori S. Prevalence of Enterocytozoon bieneusi in swine: an 18-month survey at a slaughterhouse in Massachusetts. Appl Environ Microbiol. 2002:68:2595-9.

30. Kumar S, Stecher G, Tamura K. MEGA7: Molecular Evolutionary Genetics Analysis version 7.0 for bigger datasets. Mol Biol Evol. 2016;33:1870-4.

31. Feng Y, Li N, Dearen T, Lobo ML, Matos O, Cama V, et al. Development of a multilocus sequence typing tool for high-resolution genotyping of Enterocytozoon bieneusi. Appl Environ Microbiol. 2011;77:4822-8.

32. Zhao GH, Du SZ, Wang HB, Hu XF, Deng MJ, Yu SK, et al. First report of zoonotic Cryptosporidium spp., Giardia intestinalis and Enterocytozoon bieneusi in golden takins (Budorcas taxicolor bedfordi). Infect Genet Evol. 2015:34:394-401.

33. Li W, Deng L, Yu X, Zhong Z, Wang Q, Liu X, et al. Multilocus genotypes and broad host-range of Enterocytozoon bieneusi in captive wildlife at zoological gardens in China. Parasit Vectors. 2016;9:395.

34. Wang $L$, Xiao L, Duan L, Ye J, Guo Y, Guo M, et al. Concurrent infections of Giardia duodenalis, Enterocytozoon bieneusi, and Clostridium difficile in children during a cryptosporidiosis outbreak in a pediatric hospital in China. PLoS Negl Trop Dis. 2013;7:e2437.

35. Qi M, Wang R, Wang H, Jian F, Li J, Zhao J, et al. Enterocytozoon bieneusi genotypes in grazing horses in China and their zoonotic transmission potential. J Eukaryot Microbiol. 2016:63:591-7.

36. Zhao W, Yu S, Yang Z, Zhang Y, Zhang L, Wang R, et al. Genotyping of Enterocytozoon bieneusi (Microsporidia) isolated from various birds in China. Infect Genet Evol. 2016:40:151-4. 
37. Li N, Xiao L, Wang L, Zhao S, Zhao X, Duan L, et al. Molecular surveillance of Cryptosporidium spp., Giardia duodenalis, and Enterocytozoon bieneusi by genotyping and subtyping parasites in wastewater. PLoS Negl Trop Dis. 2012;6:e1809.

38. Ben Ayed L, Yang W, Widmer G, Cama V, Ortega Y, Xiao L. Survey and genetic characterization of wastewater in Tunisia for Cryptosporidium spp., Giardia duodenalis, Enterocytozoon bieneusi, Cyclospora cayetanensis and Eimeria spp. J Water Health. 2012;10:431-44.

39. Efstratiou A, Ongerth JE, Karanis P. Waterborne transmission of protozoan parasites: review of worldwide outbreaks - an update 2011-2016. Water Res. 2017;114:14-22

40. Rosado-Garcia FM, Guerrero-Florez M, Karanis G, Hinojosa M, Karanis P. Water-borne protozoa parasites: the Latin American perspective. Int J Hyg Environ Health. 2017;220:783-98.

41. Ye J, Ji Y, Xu J, Ma K, Yang X. Zoonotic Enterocytozoon bieneusi in raw wastewater in Zhengzhou, China. Folia Parasitol (Praha). 2017;64:002.

Ready to submit your research? Choose BMC and benefit from:

- fast, convenient online submission

- thorough peer review by experienced researchers in your field

- rapid publication on acceptance

- support for research data, including large and complex data types

- gold Open Access which fosters wider collaboration and increased citations

- maximum visibility for your research: over $100 \mathrm{M}$ website views per year

At BMC, research is always in progress.

Learn more biomedcentral.com/submissions 